\title{
Death to the neutrophil! A resolution for acute respiratory distress syndrome?
}

\author{
Brittney N.V. Scott ${ }^{1,2,3}$ and Paul Kubes ${ }^{1,2,3}$
}

Affiliations: ${ }^{1}$ Dept of Physiology and Pharmacology, Cumming School of Medicine, University of Calgary, Calgary, AB, Canada. ${ }^{2}$ Dept of Critical Care Medicine, Cumming School of Medicine, University of Calgary, Calgary, AB, Canada. ${ }^{3}$ Calvin, Phoebe and Joan Snyder Institute for Chronic Diseases, Cumming School of Medicine, University of Calgary, Calgary, AB, Canada.

Correspondence: Paul Kubes, Calvin, Phoebe and Joan Snyder Institute for Chronic Diseases, 3330 Hospital Drive NW, Calgary, AB, T2N 4N1, Canada. E-mail: pkubesducalgary.ca

\section{@ERSpublications}

Neutrophil death pathways and clearance are impaired in human ARDS and interventions targeting these processes may help in the resolution of inflammation for this deadly syndrome http://ow.ly/ed2N30kW1L6

Cite this article as: Scott BNV, Kubes P. Death to the neutrophil! A resolution for acute respiratory distress syndrome? Eur Respir J 2018; 52: 1801274 [https://doi.org/10.1183/13993003.01274-2018].

Acute respiratory distress syndrome (ARDS) is a life-threatening form of respiratory failure characterised by widespread inflammatory lung injury. Damage to the delicate alveoli and microvasculature leads to significant alveolar oedema, impaired gas exchange and, ultimately, hypoxaemia [1]. ARDS can be triggered by a variety of different insults, which can be either infectious or non-infectious in nature, and common causes include pneumonia, sepsis, aspiration, noncardiogenic shock, trauma, blood transfusion and inhalation injury [1]. The initial insult leading to ARDS may directly or indirectly involve the lung (e.g. trauma to the lung versus extremities), yet a robust immune response is observed in the lung in both cases. ARDS affects $\sim 10 \%$ of patients admitted to the intensive care unit and $23 \%$ of those requiring mechanical ventilation, and has a mortality rate of $35-46 \%$ [2]. There are no therapies available for these patients and, as such, understanding the underlying mechanisms of ARDS is key to future interventional therapy.

The innate immune system plays an integral role in the pathophysiology of ARDS, but multiple views of who the culprits are exist. One view suggests that in the initial inflammatory stage, activated alveolar macrophages resident in the lung release a plethora of pro-inflammatory mediators [3]. This activates the surrounding tissue and causes other inflammatory cells, like neutrophils and monocytes, to accumulate in the microvasculature and migrate into the airways [3]. An alternative view is that a factor in blood activates the intravascular immune system, leading to the infiltration of neutrophils into the lung capillaries, adhesion and/or trapping within these small vessels, and disruption of the critical oxygen-exchanging alveolar-capillary unit. In both scenarios, activated neutrophils recruited to the lung will promote tissue injury by releasing oxidants, proteases, other inflammatory mediators and neutrophil extracellular traps (NETs) [4]. This robust inflammatory reaction leads to platelet aggregation and microthrombus formation in the lung vasculature, endothelial and epithelial cell death, loss of barrier function, and flooding of the interstitial space and alveoli [5]. While ARDS is used as an umbrella term to denote this inflammatory lung condition, whether this is one disease or multiple diseases is unknown. Nevertheless, numerous therapies aimed at suppressing or modulating inflammation for the treatment of ARDS have been investigated clinically, yet have failed to show a mortality benefit $[3,5]$. Thus, further research, particularly human research, is still needed to better understand the complex and heterogeneous immunological processes involved in the pathophysiology of ARDS. 
ARDS can be triggered by a number of different stimuli and insults; however, pneumonia- and sepsis-induced ARDS constitute the majority of cases $(\sim 60 \%$ and $\sim 15 \%$, respectively [2]) and are the most well studied. During an infection, neutrophils can release NETs (web-like structures of DNA and proteins) to help trap and kill pathogens and prevent dissemination. However, NETs can also induce significant collateral damage to surrounding tissues and have been shown to directly contribute to lung injury by inducing epithelial and endothelial cell death [6]. An interesting study by LEFrANÇAIs et al. [7] showed that a partial reduction of NETs reduced lung injury and improved survival in an animal model of bacterial acute lung injury, while complete NET inhibition increased lung bacterial load and enhanced inflammation, suggesting that a balance is needed during infection.

In order for ARDS to resolve, inflammation needs to be shut down so that reparative processes can begin and the host can recover. As inflammation subsides, barriers are re-established, fluid is reabsorbed, and cells begin to proliferate [5]. Dead or dying cells, including apoptotic neutrophils, need to be cleared and this occurs by a process called efferocytosis [8]. NETs deposited during the acute phase also need to be effectively cleared from the lung. Notably, it is thought that these mechanisms may be impaired in ARDS, leading to sustained inflammation and tissue injury and, thus, a high mortality rate as the host is unable to attain homeostasis.

It is the latter subject that was tackled in the study published in the current issue of the European Respiratory Journal by GRÉGOIRE et al. [9]. They investigated the role and clearance of neutrophils and NETs in human ARDS and offer new insights into these processes. In this study, blood and bronchoalveolar lavage (BAL) fluid samples were obtained from ARDS patients admitted to an intensive care unit in France, of which $88 \%$ of the cases were related to bacterial pneumonia and $12 \%$ were related to sepsis. The authors found that the BAL fluid of these ARDS patients had significantly increased frequencies of neutrophils, inflammatory cytokines and chemokines (i.e. interleukin (IL)-6, IL-8, CCL2 and CXCL10), and NETs (cell-free DNA) compared to the BAL fluid obtained from control patients. Moreover, the concentrations of plasminogen activator inhibitor (PAI)-1 and high-mobility group box 1 (HMGB1), which have been shown to inhibit neutrophil apoptosis [10] and promote NET release [11], respectively, in animal models of acute lung injury were also found to be elevated in the BAL fluid of the ARDS patients. Based on these results, the authors then used several different in vitro assays to further investigate NET production and clearance, as well as neutrophil apoptosis and efferocytosis.

GRÉGOIRE et al. [9] found that circulating neutrophils isolated from the ARDS patients produced significantly more NETs when stimulated with phorbol myristate acetate (PMA) compared to neutrophils obtained from healthy donors. While PMA causes lytic neutrophil NET release (referred to as NETosis) and has limited biological relevance, much more interesting was the finding that when neutrophils were exposed to BAL fluid obtained from either control or ARDS patients, only the ARDS BAL fluid stimulated NET release. This NET release is claimed by the authors to be non-lytic (i.e. vital) NETosis. While it is difficult, if not impossible, to detect lytic and non-lytic NETosis in humans, if correct this would be the first discrimination between these two types of NET release mechanisms in human disease.

The authors then examined neutrophil apoptosis and found lower proportions of apoptotic neutrophils in the circulation among the ARDS patients. Furthermore, they demonstrated that the BAL fluid of ARDS patients had the capacity to significantly reduce neutrophil apoptosis in culture. Subsequent experiments demonstrated that the capacity of macrophages to clear NETs and apoptotic neutrophils is also impaired during ARDS. Thus, this study provides new insights into human ARDS, suggesting that combined local and systemic effects of reduced neutrophil apoptosis, an increased capacity to release NETs, and decreased NET clearance and efferocytosis by macrophages contribute to the pathology of this deadly syndrome. Another clearance mechanism of neutrophils that could be impaired and is not considered by the authors is the huge number of neutrophils cleared via sputum. For example, apoptotic neutrophils may adhere less avidly to the epithelium allowing for greater clearance via sputum and, if so, reduced neutrophil apoptosis in ARDS could additionally affect this process. While often less studied than the more sexy molecular death pathways, mechanical airway clearance of neutrophils may be the dominant exit for neutrophils from lungs.

It is interesting to speculate why neutrophils migrate to the lung to induce ARDS even in situations where the injury is at arm's length from this organ. For example, major fractures, ischaemia/reperfusion of the gut or limb, and pancreatitis can all lead to subsequent ARDS and this begs the question "Why?" Recent work in a model of sterile liver injury revealed that neutrophils help heal the liver then re-enter the vasculature and go to the lung, where they are somehow told to upregulate specific receptors, including CXCR4, causing them to home back to the bone marrow to die [12]. Whether during large injuries the same thing is intended to happen and simply fails, and is overwhelmed due to the huge influx of neutrophils into the pulmonary circulation, remains to be seen. 
In a final set of experiments, GRÉGOIRE et al. [9] evaluated possible therapeutic targets to increase NET clearance and efferocytosis by the ARDS patients' monocyte-derived macrophages. They found that neutralising HMGB1 increased the clearance of apoptotic neutrophils, yet had no effect on NET uptake. Notably, however, the authors found that AMP-activated protein kinase (AMPK) activity was significantly reduced in the ARDS macrophages and that treatment with an AMPK activator, metformin, restored their phagocytic capacity to uptake both apoptotic neutrophils and NETs. AMPK is a central regulator of cellular metabolism and its activity has been shown to increase in neutrophils and macrophages during phagocytosis [13]. Thus, it is intriguing that this signalling pathway is defective during ARDS. Moreover, metformin is a widely used, clinically approved drug and these results from GRÉGOIRE et al. [9] therefore provide a compelling rationale for further investigation into its use in ARDS.

ARDS is a destructive lung syndrome with severe systemic effects. Patients who develop ARDS have a high risk of death and even those who survive are likely to suffer lifelong effects. Research into new treatment strategies for ARDS is complicated by the fact that this syndrome can be triggered by a number of different insults and that the timing of when ARDS develops after the initial insult can vary widely between patients. Moreover, clinical recognition of ARDS, particularly of less severe forms, is difficult and this probably contributes to high mortality rates even for mild ARDS [2]. It has long been known that ARDS is an inflammatory lung condition, yet research into the immunological processes that cause this syndrome is still ongoing. Animal models cannot fully recapitulate the human condition; thus, bench-to-bedside and bedside-back-to-bench approaches will be important for developing effective treatment strategies for ARDS. The current study by GRÉGOIRE et al. [9] provides some intriguing new insights into human ARDS, including a potential therapeutic target that could help in the resolution of inflammation. ARDS is a complex syndrome and further research into the mechanisms involved and new therapeutics is needed to improve its prevention and treatment.

Conflict of interest: None declared.

\section{References}

1 Fan E, Brodie D, Slutsky AS. Acute respiratory distress syndrome: advances in diagnosis and treatment. JAMA 2018; 319: 698-710.

2 Bellani G, Laffey JG, Pham T, et al. Epidemiology, patterns of care, and mortality for patients with acute respiratory distress syndrome in intensive care units in 50 countries. JAMA 2016; 315: 788-800.

3 Han S, Mallampalli RK. The acute respiratory distress syndrome: from mechanism to translation. J Immunol 2015 194: $855-860$.

4 Cheng OZ, Palaniyar N. NET balancing: a problem in inflammatory lung diseases. Front Immunol 2013; 4: 1. Thompson BT, Chambers RC, Liu KD. Acute respiratory distress syndrome. N Engl J Med 2017; 377: 562-572.

Saffarzadeh M, Juenemann C, Queisser MA, et al. Neutrophil extracellular traps directly induce epithelial and endothelial cell death: a predominant role of histones. PLoS One 2012; 7: e32366.

7 Lefrançais E, Mallavia B, Zhuo $\mathrm{H}$, et al. Maladaptive role of neutrophil extracellular traps in pathogen-induced lung injury. JCI Insight 2018; 3: e98178.

$8 \quad$ McCubbrey AL, Curtis JL. Efferocytosis and lung disease. Chest 2013; 143: 1750-1757.

9 Grégoire M, Uhel F, Lesouhaitier M, et al. Impaired efferocytosis and neutrophil extracellular trap clearance by macrophages in ARDS. Eur Respir J 2018; 52: 1702590.

10 Zmijewski JW, Bae HB, Deshane JS, et al. Inhibition of neutrophil apoptosis by PAI-1. Am J Physiol Lung Cell Mol Physiol 2011; 301: L247-L254.

11 Tadie JM, Bae HB, Jiang S, et al. HMGB1 promotes neutrophil extracellular trap formation through interactions with Toll-like receptor 4. Am J Physiol Lung Cell Mol Physiol 2013; 304: L342-L349.

12 Wang J, Hossain M, Thanabalasuriar A, et al. Visualizing the function and fate of neutrophils in sterile injury and repair. Science 2017; 358: 111-116.

13 Bae HB, Zmijewski JW, Deshane JS, et al. AMP-activated protein kinase enhances the phagocytic ability of macrophages and neutrophils. FASEB J 2011; 25: 4358-4368. 